Meta

Journal des traducteurs

Translators' Journal

\title{
Adaptation : une ambiguïté à interroger
}

\section{Yves Gambier}

Volume 37, numéro 3, septembre 1992

URI : https://id.erudit.org/iderudit/002802ar

DOI : https://doi.org/10.7202/002802ar

Aller au sommaire du numéro

Éditeur(s)

Les Presses de l'Université de Montréal

ISSN

0026-0452 (imprimé)

1492-1421 (numérique)

Découvrir la revue

Citer cet article

Gambier, Y. (1992). Adaptation : une ambiguïté à interroger. Meta, 37(3),

421-425. https://doi.org/10.7202/002802ar d'utilisation que vous pouvez consulter en ligne.

https://apropos.erudit.org/fr/usagers/politique-dutilisation/ 


\section{ADAPTATION: UNE AMBIGUIITÉ A INTERROGER}

YVES GAMBIER

Université de Turku, Turku, Finlande

Dans les références traitant de traduction, on a du mal à trouver des définitions précises, tant les concepts semblent flotter au gré des points de vue, des implicites concernant la langue, la communication, les signes, l'interculturel... Ainsi la notion d' «adaptation»: elle n'a donné lieu, semble-t-il, à aucune analyse rigoureuse, systématique; on en trouve des traces, des explications parcellaires, surtout dans les réflexions sur la traduction littéraire... Je me propose ici d'interroger ce manque, ce qu'il peut signifier: il apparaîtra que les études en traduction, prétendument théoriques, sont encore largement marquées par l'idéologie, les jugements de valeur. Une traductologie est-elle possible avec une telle prégnance de l'opinion?

\section{REMARQUES GÉNÉRALES}

1. Quelle est la pertinence des deux termes traduction et adaptation? Bien que souvent co-présents, ils ne s'autodélimitent pas clairement: on les rapproche mais sans préciser leur frontière, sinon leur relation. Rattachée à certains types de texte (pièces de théâtre, publicité par exemple), l'«adaptation» semble impliquer une certaine liberté du traducteur - à qui il serait alors permis des modifications, des ajouts, des ajustements, des omissions... au texte de départ, pour mieux le plier aux récepteurs visés (spectateurs, consommateurs), à leurs habitudes et à leurs normes de réception. Certains parlent même dans ce cas de «pseudotraduction». Implicitement, la «traduction» se définirait donc comme un effort littéral, une mimesis de l'original.

2. Ainsi, dans la plupart des cas, on se retrouve devant une antinomie intenable: d'une part, la traduction serait vouée à la littéralité, d'autre part, elle se changerait en «adaptation» dès que son souci cibliste dominerait. L'alternative n'est pas nouvelle; elle reflète une autre dichotomie touchant la communication:

- celle qui ne marcherait que prise au pied de la lettre, sans jeu ni ambiguïté, sinon le malentendu devient la norme, l'échange se transmue en non-communication; la communication ici est synonyme de transaction consensuelle, coopérative, fortement ritualisée, codée, où chacun reste à sa place, joue son rôle dans des limites imparties;

- celle dynamique qui tente de tenir compte des intentions des interlocuteurs, de leurs motivations, de leurs intérêts... La communication est interaction, travail de co-production, transformation d'énergie où les pertes informationnelles, les blessures symboliques sont compensées par le gain, c'est-à-dire l'ouverture infinie d'un espace de communicabilité : l'échange peut avoir des ratés, il permet sans cesse sa reprise. Ces ratés, ces pertes sont à la fois la limite et le dépassement de l'interaction: dans la dialectique interactionnelle, ils ne se figent pas en silence, en mutisme.

3. Le concept de traduction ne peut se borner bien sûr au mot à mot: dans la vie professionnelle, on constate en effet que tous les textes traduits ne sont pas forcément identifiés comme traduction - par exemple, dans les médias, que les traductions ne 
recouvrent pas toujours des textes entiers (traductions sélectives, abrégées) ni même des textes à rédaction soignée (traductions automatiques sans post-édition, suffisantes pour des ingénieurs, des scientifiques à la recherche d'une information «adaptée» à leurs besoins d'accès rapide à des données ponctuelles).

Le même flou conceptuel vaut pour l'adaptation - doublé ici du flou terminologique, puisque l'adaptation trouve comme synonymes: traduction libre, oblique, transposition, arrangement... On peut dégager au moins trois sens - sans parler du processus social, culturel qui consiste à se rendre conforme aux normes dominantes, à s'harmoniser avec elles, à s'intégrer à un nouveau réseau de solidarité, de valeurs (exemple s'adapter à un nouveau voisinage ; l'expatrié s'adapte, ou pas, à son pays d'accueil...):

a) Ajouter et/ou retrancher pour que le texte d'arrivée (TA) ait le «même effet» que le texte de départ (TD), l'accent étant mis sur les récepteurs (culture et langue d'arrivée): c'est la traduction dynamique de Nida, la traduction communicative de Newmark, la traduction déguisée (covert translation) de J. House (1977)... Dans ce cas, la traduction qui approche de la différence, de l'autre... fait disparaître la différence, réduit l'autre au même (à soi): elle fait comme si elle était l'original, déliée de son contexte de départ; tout en prétendant «conserver» la fonction de TD en TA, elle «modifie» les présuppositions du texte pour les «adapter» aux nouvelles conditions de réception. La contradiction des termes ici éclaire les hésitations courantes entre traduction et adaptation.

Dans cette perspective, on citera en exemple les «belles infidèles» - fréquentes aux $\mathrm{XVII}^{\mathrm{e}}$ et $\mathrm{XVIII}{ }^{\mathrm{e}}$ siècles en France mais sans être limitées à cette période ni formes exclusives d'alors, les textes TD édulcorés, censurés pour être restitués selon des canons acceptables en TA - exemple de Lucrèce, de Catulle rendus en français, exemple de l'Énéide de Virgile traduit par du Bellay $\left(\mathrm{XVI}^{\mathrm{e}}\right)$, par Klossowski, D. Robert $\left(\mathrm{XX}^{\mathrm{e}}\right)$.

b) Faire auvre originale, à partir d'une autre composée dans le même système de signes ou pas - c'est alors la traduction sémiotique selon R. Jakobson (1967): cas d'un poème mis en chanson, d'un roman «adapté» en film, d'une pièce de théâtre «librement adaptée» pour la télévision, de la «transposition» de Ch. Dickens, du Coran... en BD... Ces «adaptations» peuvent se faire parfois avec traduction interlinguistique: par exemple Andromaque d'Euripide «repris» par Racine, les Fables de La Fontaine, «inspirées» des récits du grec Ésope, du latin Phèdre... - la relecture de la préface de l'édition de 1668 et l'Avertissement du Second Recueil (1678) permet d'ailleurs de mesurer combien l'imitation prétendue est enrichissement, invention, modification, forçant ainsi à s'interroger sur les interférences entre plagiat, pastiche, parodie... et adaptation! C'est à ce sens (b) que s'applique exclusivement la dénomination d'adaptateur.

c) Transformer un texte en vue d'un certain lectorat, pour des raisons et selon des critères socio-économiques déclarés ou pas: adaptation par exemple de Montaigne pour tel club de livres, de Camus en «français facile» pour les étrangers, reformulation délibérée par M. Tournier de Vendredi ou les Limbes du Pacifique (1967) en une version pour jeunes lecteurs (Vendredi ou la vie sauvage, 1971), «transposition» en québécois (langue et contexte) du Bourgeois gentilhomme de Molière par Antonine Maillet, etc.

Cette adaptation-réécriture (avec substitution de mots, choix de génériques, condensation de syntagmes, paraphrasage, contraction de paragraphes, modification de temps, manipulation de propositions complexes, nouvel agencement thématique, raccourcissement de phrases...) est une forme de traduction intralinguale (Jakobson 1967), non sans analogie avec le parler de l'adulte bavardant avec un petit enfant, le parler d'un natif s'adressant à un non-natif (migrant, public d'étrangers...), non sans analogie avec la 
vulgarisation scientifique, la propagande politique, les campagnes publicitaires... supposées viser des publics précis.

En bref, non seulement la division traduction-adaptation reste ambiguë mais les notions mêmes de traduction et d'adaptation sont polymorphes - ceci expliquant peutêtre cela.

\section{EXEMPLES}

Afin de mieux préciser, si possible, l'«adaptation», il vaut la peine encore de s'attarder sur quelques exemples portant une telle étiquette - notamment dans les sens (a) et (c) esquissés ci-dessus.

Ainsi paraissent aujourd'hui en langue contemporaine des textes d'ancien et de moyen français (Villon, Chrétien de Troyes...), pour le plaisir de lire des ceuvres du patrimoine, le déplaisir (?) du déchiffrage de mots désuets, de formes latinisantes étant l'apanage d'une minorité de plus en plus réduite sans doute... Montaigne auteur du XXe siècle, alors? Rabelais vient de sortir en finnois de $1990 \ldots$.. À quel niveau du texte se fait cette actualisation linguistique? Jusqu'où est-elle légitime? À partir de quand peut-on dire qu'elle défigure, «trahit» l'original ? Selon quelles normes se réalise-t-elle ? Cette traduction intralinguale est-elle adaptation contrainte? Si oui, quelles sont ces contraintes (linguistiques et extra-linguistiques)? Quel rapport entretient-elle avec la retraduction?

- Ainsi l'«adaptation» de l'édition chinoise en 10 volumes de l'Encyclopadia Britannica (1986): cette transposition culturelle et politique confinant à la censure (suppressions pures et simples de certaines rubriques, reformulation d'autres - par exemple sur Taïwan, sur le stalinisme...).

- Ainsi la traduction des livres pour enfants, accompagnée souvent d'explications, de notes, faite de substitutions, de simplifications, d'abrègements, d'omissions, de modernisations... sans parler de réécritures parfois. L'adaptation, délibérée ici, reconnue, de l'arrière-plan culturel, des images, des noms propres... peut s'étendre jusqu'à l'adaptation au goût du jour, à une moralisation nouvelle. La vingtaine de versions anglaises du Vilain petit canard d'Andersen tendent par exemple à remplacer par un problème alimentaire tout ce qui touche au sexe, la morale danoise finale est omise ou réduite (Pedersen 1990); dans tel récit suédois, le besoin de faire pipi se transforme en besoin de promenade dans une version allemande... L'effacement des différences culturelles - pour faire comme si la traduction était l'original sur une structure narrative presque inchangée, est justifié pour ne pas dépayser les jeunes lecteurs : à quoi sert dans ces conditions la traduction qui annule les différences, les distances? Une telle adaptation-distorsion est un autre nom de la censure; substitut d'un original considéré comme trop étranger, trop osé, trop exotique... elle se veut compensation. Il y a forte connivence ici avec certaines façons de traiter la littérature enfantine comme secondaire, forme affadie de la «grande» littérature.

- Ainsi la Bible rendue dans des versions plus ou moins réputées (versions King James, de Douai, de Le Maistre de Saci, de l'Alliance biblique universelle, la TOB ou traduction œcuménique de la Bible...). Tantôt ces versions se déclarent traduction selon l'esprit du texte, «adaptée» à la sensibilité culturelle locale (perspective théorisée entre autres par Nida en 1964 et sa proposition d' «équivalence dynamique»); tantôt elles clament leur attachement à la lettre, aux rouleaux anciens (versions dites de Chouraqui, de Meschonnic...). On retrouve les tensions, sinon les contradictions, exprimées depuis Saint Jérôme, entre les options de communication et de littéralité - cette dernière se légitimant sous prétexte que la «parole» sacrée est mystère, intouchable. 
Ainsi les textes philosophiques et autres discours culturels théoriques: en sciences sociales, en anthropologie, en sciences politiques, les textes freudiens..., plus ou moins marqués par la tradition d'exégèse. La «traduction» est un point aveugle pour leurs lecteurs éventuels qui n'ont pourtant pour la plupart accès à ces textes qu'à travers la «traduction»: Kant lu-rendu par Tremesaygues, par Barni ou par Philonenko, Hegel lurendu par J. Hyppolite, Heidegger lu-rendu par Corbin, par Martineau... Là aussi, la retraduction n'est jamais sans effet ni enjeu - qu'on pense aux retraductions en français de Freud, de Platon, de Leibniz, en anglais de Descartes... faites à la suite de nouvelles interrogations lacanienne, herméneutique, épistémologique...; qu'on pense également aux (re)traductions via une langue pivot, une double interprétation (Bourdieu, Lyotard en finnois à partir de l'anglais...).

Traduction libre, modification, actualisation, distorsion... décidément, c'est toujours par rapport à autre chose que l'adaptation essaie de se définir (rapport à des normes linguistiques, des conventions stylistiques, des schémas de communication, des valeurs acceptables...). L'histoire de la traduction serait-elle l'histoire d'un malentendu permanent, d'un manque constant, d'une perte obsessionnelle... qui ne dirait son nom que parfois en se reconnaissant comme «adaptation»?

On peut affirmer deux choses à la suite de ces remarques et exemples:

traduction/adaptation: il s'agit d'une taxinomie binaire qui présuppose une certaine fétichisation du texte TD; elle est hâtive et antinomique comme souvent en traduction (littéraire/non littéraire, littérale/libre, forme/contenu...), c'est-à-dire qu'elle nie tout degré de transfert dans le processus traductionnel.

\section{DE LA TRADAPTATION}

La traduction, comme toute autre forme de communication, est médiation, c'est-àdire ajustement à un contexte, à certaines visées ou intentions, à des lecteurs... à la fois réels et objets de représentations, de fantasmes. Elle est travail, négociation de sens, interaction : elle est forcément adaptation, comme toute communication, et non pure translation de formes. Traduire un mode d'emploi, une brochure touristique, un poème... ne relève pas d'une antinomie tranchée - avec d'un côté une «adaptation» qui serait... libre et de l'autre une littéralité... qui serait stricte. Cette alternative, ce dilemme n'éclaire pas du tout la traduction, comme processus et comme produit. Toute traduction - qu'elle soit étiquetée comme «adaptation» ou qu'elle soit adaptation non reconnue, honteuseest activité de reformulation nécessairement - en vue de réaliser certains objectifs, d'atteindre certains buts. Pour un même but, il y a ainsi des parties «traduites» (littéralement) et des parties «adaptées» (par rapport aux conditions de réception en LA).

L'«adaptation» - un des procédés de traduction définis par Vinay et Darbelnet concourt au résultat final, à savoir le TA. Mais les autres modes (condensation, omission...) sont aussi formes d'adaptation!

En résumé: 1) l'opposition traduction/adaptation véhicule une opposition implicite entre littéralité (accent sur TD) et liberté (accent sur TA) qui relève d'un jugement de valeur, bien plus que d'une explication raisonnée du transfert entre TD et TA;

2) l'adaptation est à la base même du processus de communication, compris comme ensemble de stratégies, de procédés pour construire, échanger du sens. Les deux significations d'《adaptation» ne sont pas inconciliables: la communication comme ajustement à divers paramètres situationnels, extra-linguistiques et linguistiques présuppose une liberté de choix dans ses moyens. 
Un autre paradoxe marque l'utilisation de l'étiquette «adaptation» par rapport à celle de «traduction»: on parlerait en effet d'adaptation au-delà d'un certain seuil quand le nombre et le type de transformations du TD sont tels qu'il y a réécriture, assimilation aux normes, aux conventions, aux valeurs de la langue-culture réceptrice - d'où la fréquence du terme pour les textes dramatiques, publicitaires, pour enfants... La «liberté» du traducteur se réduirait à coloniser le TD (adaptation assimilatrice). Or, ne diton pas le plus souvent qu'une traduction «réussie» est celle qui ne se donne pas à voir, qui ne montre pas le travail de transfert, qui fait comme si elle était un original (cf. I.3.a)? En toute logique, le discours dominant en théorie de la traduction devrait donc valoriser... l'«adaptation»!

La paire traduction/adaptation - avec fétichisation tantôt du TD tantôt des règles en LA - signale donc une contradiction de l'idéologie traductionnelle courante: on devrait rechercher l'«équivalence dynamique» (atteindre aux mêmes effets en LA qu'en LD, dépasser le transcodage, par conséquent reconnaître les ajustements nécessaires) et en même temps ne pas occulter le travail du traducteur. Peut-on à la fois faire comme si on était l'original, c'est-à-dire effacer les traces du transfert, et revendiquer une place pour la signature du traducteur, c'est-à-dire manifester le passage qui a eu lieu?

Pour dépasser l'opposition et la contradiction, le concept et le terme de TRADAPTATION, proposé par le Québécois Michel Garneau (cf. Delisle 1986), semble pertinent non pas seulement pour le théâtre mais pour tous les textes à rendre en LA. Il permet d'affirmer que toute traduction est adaptation; cependant:

1) toutes les parties d'un texte TD n'atteignent pas le même degré d'adaptation: il y a des adaptations stratégiques - on adapte à une nouvelle situation, on élabore un nouvel équilibre entre auteur et destinataires...; il y a des adaptations tactiques, au niveau linguistique (jeux de mots, registres de langue, idiotismes...) et au niveau extra-linguistique (référence à des traditions locales, à des institutions, termes dits culturels...);

2) tous les types de texte n'appellent pas le même degré d'adaptation, n'autorisent pas la même liberté d'action de la part du traducteur - médiateur non pas tant comme individu que comme acteur social répondant aux exigences collectives contingentes: les attentes envers un article en physique, un contrat commercial ne sont pas identiques à l'heure actuelle aux attentes envers une chanson, un sous-titrage...

\section{BIBLIOGRAPHIE}

DELISLE, J. (1986) : «Dans les coulisses de l'adaptation thêâtrale», Circuit 12, pp. 3-8.

HOUSE, J. (1977): A Model for Translation Quality Assessment, Tübingen, Narr.

JAKOBSON, R. (1967): «On Linguistic Aspects of Translation», On Translation, R.A. Brower (Ed.), Cambridge, Mass., Harvard University Press.

Meta (1972) : «L'adaptation publicitaire», numéro spécial 17-1, Presses de l'Université de Montréal.

NIDA, E. (1964): Toward a Science of Translation, Leiden, E.J. Brill.

PEDERSEN, V. H. (1988): Essays on Translation, Copenhague, Nyt Nordisk forlag.

PEDERSEN, V. H. (1990): «Ugly Ducklings?», Proceedings from SSOTT III: Translation Theory in Scandinavia, 11-13 août 1988, pp. 229-242, Université d'Oslo.

ST (Société française des traducteurs) (1988): La traduction face aux nouveaux médias (l'adaptation publicitaire), Journée organisée par la SFT lors d'Expolangues, 20 février 1988, broché, $34 \mathrm{p}$.

ZUBER, O. (Ed.) (1980): The Languages of Theatre: Problems in the Translation and Transposition of Drama, Oxford, Pergamon Press.

ZUBER-SKERRITT, O. (1984): Page to Page: Theatre as Translation, Amsterdam, Rodopi. 